\title{
Bridge to second double lung transplant with an extracorporeal carbon dioxide removal system in sites inversus patient
}

\author{
Masashi Furukawa ${ }^{1}$, Ernest Chan $^{1}$, and Pablo Sanchez ${ }^{1}$ \\ ${ }^{1}$ University of Pittsburgh Department of Cardiothoracic Surgery
}

February 20, 2022

\begin{abstract}
We report a first case with the use of extracorporeal carbon dioxide removal system as a bridge to re-do lung transplant in complete situs inversus patient. A 29-year-old female with Kartagener syndrome and complete situs inversus underwent a double lung transplant for end stage lung disease. Within one year after transplant the patient had primarily hypercapnic respiratory failure with radiographic signs of chronic lung allograft dysfunction. To optimize her nutritional status and muscle strength before re-do lung transplantation, we decided to bridge her with an extracorporeal carbon dioxide removal system due to anatomical difficulty. She was listed and underwent an uneventful re-do double lung transplant with cardiopulmonary support.
\end{abstract}

\section{INTRODUCTION}

The use of extracorporeal life support (ECLS) as a bridge to lung transplantation has increased over the last decade, however its use in re-do lung transplantation is limited due to poor outcomes ${ }^{1,2}$. Extracorporeal circulation with a single duo-lumen cannula provides the advantage of more comfortable mobilization particularly in patients in which we expect a longer bridge to transplant. The Hemolung Respiratory Assisted System (HRAS) (ALung Technologies, Pittsburgh PA) is a venous-venous extracorporeal carbon dioxide removal $\left(\mathrm{ECCO}_{2} \mathrm{R}\right)$ system that allows for effective removal of carbon dioxide via a small French double lumen catheter ${ }^{2,3}$.

In this report the decision to bridge our patient with an HRAS was based on primary hypercapnic respiratory failure and anatomical limitations due to her complete situs inversus.

\section{CASE PRESENTATION}

A 29-year-old female with Kartagener syndrome and complete situs inversus underwent a double lung transplant for end stage lung disease. Her postoperative course was uneventful. Within 6 months of transplant, she experienced an aspiration event leading to a persistent decline in her lung function within the first year. She ultimately was hospitalized requiring mechanical ventilation and a tracheostomy. The patient had primarily hypercapnic respiratory failure with radiographic signs of chronic lung allograft dysfunction (bronchitis obliterans syndrome). At the time of admission, the patient ventilatory rate was 30 breaths $/ \mathrm{min}$ and her arterial carbon dioxide tension $\left(\mathrm{PaCO}_{2}\right)$ was $80 \mathrm{mmHg}$. Her increased respiratory work resulted in high calorie consumption with a significant impact on her body mass index (16.5 to 14.4 in 6 months). To optimize her nutritional status and muscle strength before re-do lung transplantation, we decided to bridge her with extracorporeal membrane oxygenation (ECMO). Initially, our intention was to use a dual-lumen cannula to facilitate mobility but due to her anatomy we decided that an Avalon or Crescent cannula was not suitable as the outflow jet will be directed against the atrial wall. We decided to proceed with the HRAS after emergency FDA authorization. A dual-lumen $15.5 \mathrm{Fr}$ catheter was placed via the left internal jugular vein into the superior vena cava (Figure 1A, 1B). After the initiation of $\mathrm{ECCO}_{2} \mathrm{R}$, the patient's $\mathrm{PaCO}_{2}$ improved 
by adjusting sweep between 7-9 L/min resulting in a carbon dioxide $\left(\mathrm{CO}_{2}\right)$ removal of $80-90 \mathrm{ml} / \mathrm{min}$ (Figure 2). We used bivalirudin for anticoagulation and targeted goal was activated partial thrombin time 45-60 s. Three days after placing the HRAS, she was listed for re-do double lung transplantation. As she waited for a viable donor offer, she was awake, able to get out of bed, stand up and actively participate in rehabilitation program. In addition, she stopped losing weight, her BMI plateau at 15 and her prealbumin at $20 \mathrm{mg} / \mathrm{dl}$. Donor lungs became available 8 days after the initiation support. After induction of general anesthesia, the HRAS was removed after confirming that the patient was hemodynamically stable, and cardiopulmonary bypass was introduced when the donor lungs arrived in the operating room. She underwent an uneventful re-do double lung transplant. After transplantation, the patient developed an increased need for oxygen, potentially due to extensive volume and product resuscitation and ischemic reperfusion injury. We decided to put her on venous-venous ECMO (VV-ECMO). Her chest was left open due to coagulopathy for 3 days. We decannulated VV-ECMO on postoperative day 7. She was discharged 43 days after lung transplantation (Figure 1C).

\section{DISCUSSION}

Lung transplantation in patients with Kartagener's syndrome and situs inversus challenges include: dextrocardia with discrepancies between donor and recipient vessels and airways ${ }^{4-7}$. In addition, the chest cavity mismatch between the donor right lung between and the recipient's dextrocardia can result in right lower lobe atelectasis and kinks of the pulmonary artery. In the present report we did not experience any anastomotic or postoperative challenges. We only preserved donor pulmonary arteries to remain long enough for anastomosis.

We previously reported a case of using HRAS to bridge re-do double lung transplant; transplant was performed 20 days after the induction of $\mathrm{HRAS}^{2}$. Bonnin et al. reported using HRAS for severe chronic obstructive pulmonary disease bridge to lung transplant; transplant was performed 31 days after induction of HRAS $^{3}$. HRAS has also been reported to improve acidosis in acute respiratory failure from coronavirus disease $2019^{8}$.

Rajab et al. reported the use of VV-ECMO via a right internal jugular Avalon cannula as a bridge to heart and lung transplantation in a patient with dextrocardia ${ }^{9}$. In our patient this was not an option because her superior and inferior vena cava were on the left side and her tricuspid valve was facing right. We decided to proceed with the Hemolung because our patient mainly required $\mathrm{CO}_{2}$ removal to reduce ventilatory need, reduce work of breathing and reduce calory consumption. In addition, the $15.5 \mathrm{Fr}$ was easy to place inside the right atrium via the left internal jugular vein.

This experience demonstrates that $\mathrm{ECCO}_{2} \mathrm{R}$ can be safely and effectively used to bridge lung transplant patients with hypercapnic respiratory failure in which anatomical challenges limit cannulation options. To our knowledge this may represent the first experience with the use of $\mathrm{ECCO}_{2} \mathrm{R}$ as a bridge to lung retransplantation in a patient with complete situs inversus.

\section{References}

1. Tipograf Y, Salna M, Minko E, et al. Outcomes of Extracorporeal Membrane Oxygenation as a Bridge to Lung Transplantation. Ann Thorac Surg. 2019;107(5):1456-1463.

2. Bermudez CA, Zaldonis D, Fan MH, Pilewski JM, Crespo MM. Prolonged Use of the Hemolung Respiratory Assist System as a Bridge to Redo Lung Transplantation. Ann Thorac Surg. 2015;100(6):2330-2333.

3. Bonin F, Sommerwerck U, Lund LW, Teschler H. Avoidance of intubation during acute exacerbation of chronic obstructive pulmonary disease for a lung transplant candidate using extracorporeal carbon dioxide removal with the Hemolung. J Thorac Cardiovasc Surg. 2013;145(5):e43-44.

4. Yamamoto H, Sugimoto S, Miyoshi K, Otani S, Yamane M, Toyooka S. Lung transplantation for Kartagener syndrome: technical aspects and morphological adaptation of the transplanted lungs. Gen Thorac Cardiovasc Surg. 2021;69(3):588-592. 
5. Lucius Lee KF, Michael Hsin KY, Alva Sit KY, Cally Ho KL, Timmy Au WK. Bilateral sequential lung transplantation in Kartagener syndrome.JTCVS Tech. 2020;3:406-408.

6. de Castro CCB, Dos Reis FP, de Carvalho GVS, et al. Technical Challenges in Lung Transplantation of Kartagener Syndrome Recipients: A Unique Team Experience With 12 Patients. Transplant Proc.2020;52(5):1384-1387.

7. Gauthier JM, Takahashi T, Bierhals AJ, et al. Technical Considerations for Lung Transplantation in Kartagener's Syndrome.Ann Thorac Surg. 2019;107(5):e337-e339.

8. Akkanti B, Jagpal S, Darwish R, et al. Physiologic Improvement in Respiratory Acidosis Using Extracorporeal Co2 Removal With Hemolung Respiratory Assist System in the Management of Severe Respiratory Failure From Coronavirus Disease 2019. Crit Care Explor.2021;3(3):e0372.

9. Rajab TK, Kim T, Keller S, Mallidi H. Management of a young patient with dextrocardia, atrial septal defect, and Eisenmenger syndrome with venous-venous extracorporeal membrane oxygenation and heart-lung transplantation. J Card Surg. 2019;34(10):1114-1116.

Figure 1, A: Hemolung, B: X-ray with Hemolung, C: X-ray at discharge

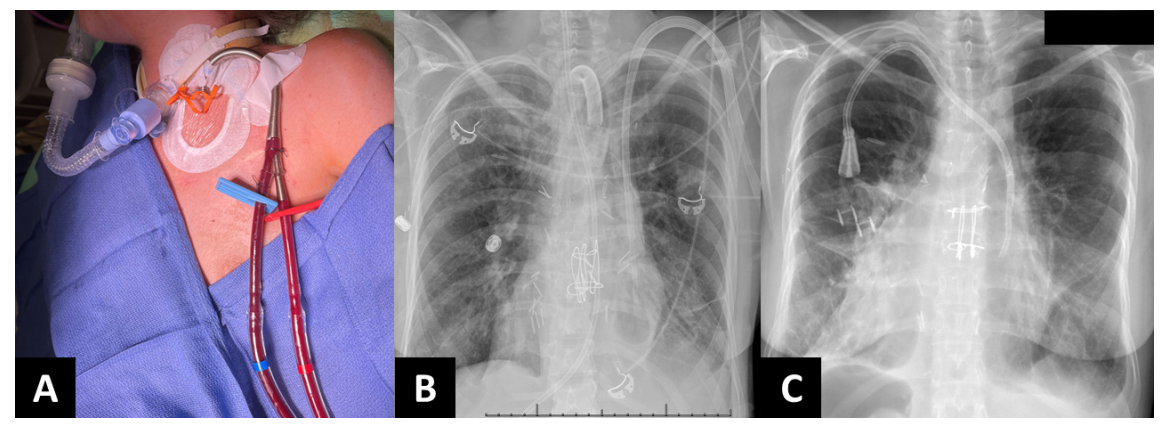

Figure 2:

Top: CO2 removal and sweep gas flow during 8 days of Hemolung support.

Bottom: Patient's PaCO2 during 8 days of Hemolung support. 

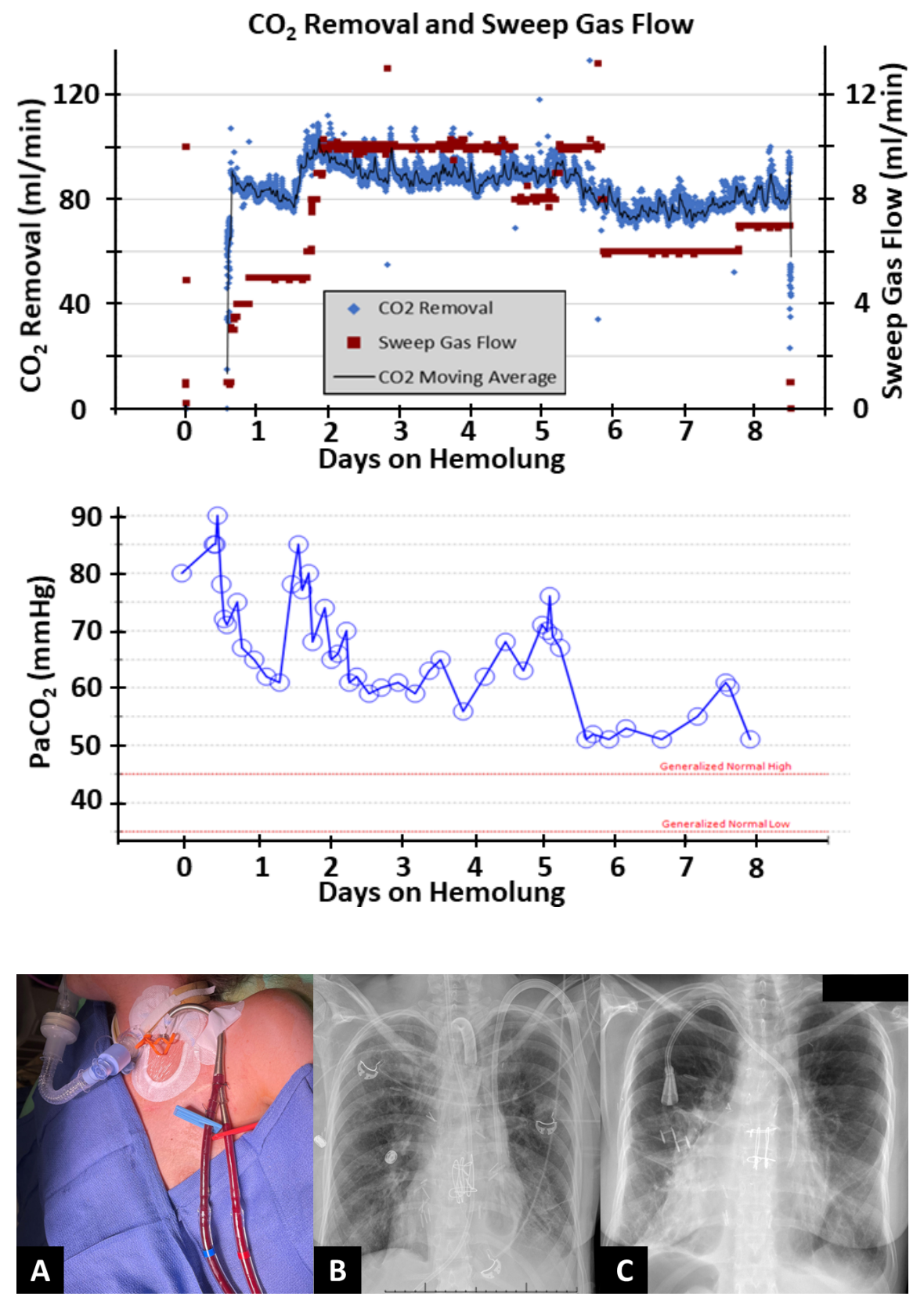

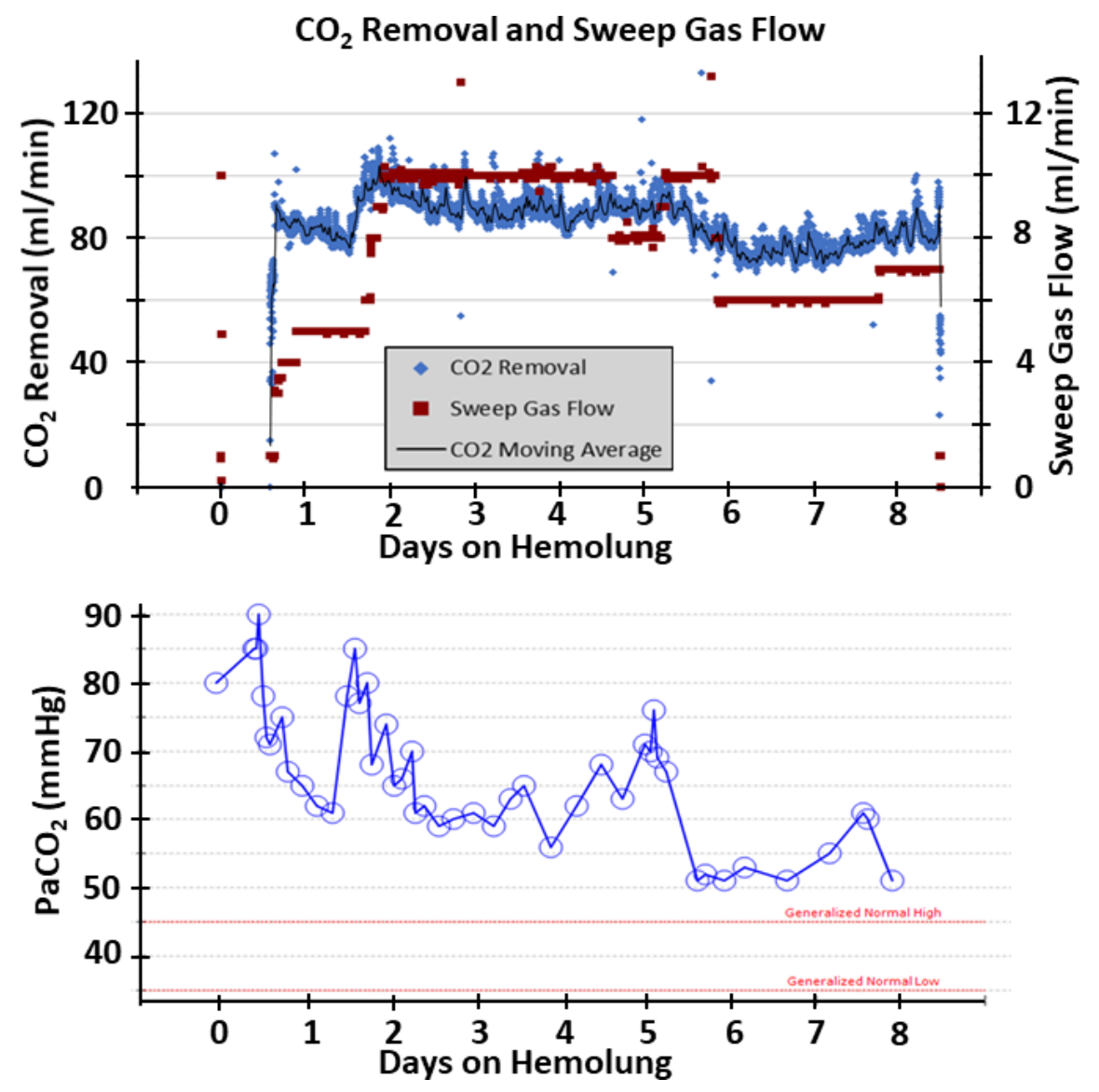